\title{
PETTIS INTEGRABILITY OF FUZZY MAPPINGS WITH VALUES IN ARBITRARY BANACH SPACES
}

\author{
L. DI PIAZZA AND V. MARRAFFA
}

\begin{abstract}
In this paper we study the Pettis integral of fuzzy mappings in arbitrary Banach spaces. We present some properties of the Pettis integral of fuzzy mappings and we give conditions under which a scalarly integrable fuzzy mapping is Pettis integrable.

Cordially dedicated to Professor Paolo de Lucia

on the occasion of his 80-th birthday with esteem and admiration
\end{abstract}

\section{INTRODUCTION}

Due to interest in applications (e.g. control theory, optimization or mathematical economics), a wide theory was developed for set-valued integrability in separable Banach spaces (see [1, 6], 18, and reference inside, [12, 13, [15, [20]). Recently several authors studied set-valued integrability without separability assumptions in Banach spaces (see [2, 3], [5, 7, 9, , 10, 14] and 22]). It is well known the useful role played by fuzzy mappings in the theory of fuzzy sets and their applications. Integrals of fuzzy mappings are a natural extension of set-valued integrability. For fuzzy mappings in $\mathbb{R}^{n}$ an integral was introduced in [19] and 21, while the case of fuzzy mappings in separable Banach space was considered and studied in [32, 33] and 34. The fuzzy Henstock integral of fuzzy mappings in $\mathbb{R}$, or $\mathbb{R}^{n}$, has been introduced and studied in [27, 28] and [4, while in [23] the more general case of Banach space valued fuzzy Henstock integral is considered.

Park in 24] introduced the Pettis integral for fuzzy mappings in separable Banach spaces.

In this paper we continue the study of the Pettis integral for fuzzy mappings in not necessarily separable Banach spaces. An important technical tool for our investigation is the following decomposition result (Theorem 3.4)

2010 Mathematics Subject Classification. Primary 26E50; Secondary 28E10, 03E72.

Key words and phrases. Fuzzy Pettis integral, generalized fuzzy number measure, fuzzy weak integrability.

The authors were partially supported by the grant of GNAMPA prot. U 2016/000386 . 
$\widetilde{\Gamma}: \Omega \rightarrow \mathcal{F}(X)$ (here $\mathcal{F}(X)$ is the generalized fuzzy number space associated to the Banach space $X)$ is a Pettis integrable fuzzy mapping if and only if $\widetilde{\Gamma}$ can be represented as $\widetilde{\Gamma}(\omega)=\widetilde{G}(\omega)+\widetilde{f}(\omega)$, where $\widetilde{G}: \Omega \rightarrow \mathcal{F}(X)$ is a Pettis integrable fuzzy mapping whose support functions are nonnegative and $\widetilde{f}$ is a Pettis integrable fuzzy mapping generated by a Pettis integrable selection of $\widetilde{\Gamma}$.

In [22] (pretty level-wise) results regarding the Pettis integrability of scalarly integrable multifunction are proved. Here it is shown a kind of their "consistency" with respect to the fuzzy nature of the fuzzy Pettis integral. Indeed, we prove that the primitive of a Pettis integrable fuzzy mapping is a generalized fuzzy number measure (Theorem 3.5) and we give a characterization of the Pettis integrable fuzzy mappings by means the notion of core (Theorem 4.1).

It is well known that if $X$ is a separable Banach space and $c_{0} \nsubseteq X X$, then each single $X$-valued scalarly integrable function is Pettis integrable. An analogue result, for weakly integrable fuzzy mappings has been proven in [32] under the hypothesis that the target Banach space is separable. In Theorem 3.8 and Theorem 4.2 we present non-separable versions of the above result.

\section{BASIC FACTS.}

Let $(\Omega, \mathcal{L}, \mu)$ be a measure space, where $\mathcal{L}$ is the family of all $\mu$-measurable sets. Let $X$ be a Banach space endowed with the norm $\|\cdot\|$ and let $X^{*}$ be its dual, $B(X)$ and $B\left(X^{*}\right)$ will denote respectively the unit ball in $X$ and in $X^{*}$. The symbol $c(X)$ stands for the family of all nonempty closed convex subsets of $X$, while $c w k(X)$ denotes the subfamily consisting of weakly compact sets. In $\operatorname{cwk}(X)$ we consider the Minkowski addition $(A+B:=\{x+y: x \in A, y \in B\})$ and the standard multiplication by scalars $(k A:=\{k x: x \in A\})$. If $A \in \operatorname{cwk}(X)$ and $x \in X$, by the symbol $A-x$ we denote the set $\{y \in X: y=z-x, z \in A\}$. Moreover we endow $c w k(X)$ with the Hausdorff distance

$$
d_{H}(A, B):=\max \left\{\sup _{x \in A} \inf _{y \in B}\|x-y\|, \sup _{y \in B} \inf _{x \in A}\|x-y\|\right\} .
$$

The space $c w k(X)$ with the Hausdorff distance is a complete metric space. For every $A \in \operatorname{cwk}(X)$ the support function of $A$ is denoted by $s(\cdot, A)$ and defined by $s\left(x^{*}, A\right):=\sup \left\{\left\langle x^{*}, y\right\rangle: y \in A\right\}$, for each $x^{*} \in X^{*}$. Clearly the map $x^{*} \longmapsto s\left(x^{*}, A\right)$ is sublinear on $X$ and $-s\left(-x^{*}, A\right)=\inf \left\{\left\langle x^{*}, y\right\rangle: y \in A\right\}$, for each $x^{*} \in X^{*}$.

Below, by a series $\sum_{n=1}^{\infty} A_{n}$, where $A_{n} \in 2^{X} \backslash\{\emptyset\}$ for each $n$, we understand the set

$$
\left\{\sum_{n=1}^{\infty} x_{n}: x_{n} \in A_{n} \text { and } \sum_{n=1}^{\infty} x_{n} \text { unconditionally convergent }\right\} .
$$


Let $u: X \rightarrow[0,1]$. We set $[u]^{r}=\{x \in X: u(x) \geq r\}$, for $r \in(0,1] ; u$ is called a generalized fuzzy number, as in [32] and [33], (fuzzy number as in [31) on $X$ if, for each $r \in(0,1],[u]^{r} \in \operatorname{cwk}(X)$. Let $\mathcal{F}(X)$ denote the generalized fuzzy number space.

We define $\theta: X \rightarrow[0,1]$ as follows:

$$
\theta(x)=\left\{\begin{array}{lll}
1 & \text { if } & x=0 \\
0 & \text { if } & x \neq 0
\end{array}\right.
$$

Then $\theta \in \mathcal{F}(X)$ and $\theta$ is called the null element of $\mathcal{F}(X)$.

In the sequel we will use the following representation theorem (see [31]).

Theorem 2.1. If $u \in \mathcal{F}(X)$, then

(i) $[u]^{r} \in \operatorname{cwk}(X)$, for all $r \in(0,1]$;

(ii) $[u]^{r_{2}} \subseteq[u]^{r_{1}}$, for $0 \leq r_{1} \leq r_{2} \leq 1$;

(iii) if $\left(r_{k}\right)$ is a nondecreasing sequence converging to $r>0$, then

$$
[u]^{r}=\bigcap_{k \geq 1}[u]^{r_{k}} .
$$

Conversely, if $\left\{A_{r}: r \in(0,1]\right\}$ is a family of subsets of $X$ satisfying $(i)-($ iii $)$, then there exists a unique $u \in \mathcal{F}(X)$ such that $[u]^{r}=A_{r}$ for $r \in(0,1]$.

For $u, v \in \mathcal{F}(X)$ and $k \in \mathbb{R}$, define $u+v$ and $\lambda u$ as follows (see [24, pag. 536])

$$
\begin{gathered}
(u+v)(x)=\sup _{x=y+z} \min (u(y), v(z)), \\
(\lambda u)(x)= \begin{cases}u\left(\frac{1}{\lambda} x\right) & \text { if } \lambda \neq 0 \\
\widetilde{0} & \text { if } \quad \lambda=0, \quad \text { where } \widetilde{0}=\chi_{\{0\}}\end{cases}
\end{gathered}
$$

For $u, v \in \mathcal{F}(X)$ and $k \in \mathbb{R}$, the addition and the scalar multiplication are defined respectively by

$$
[u+v]^{r}:=[u]^{r}+[v]^{r} \text { and }[k u]^{r}:=k[u]^{r},
$$

for each $r \in(0,1]$.

Definition 2.2. (see [33, Definition 3.1]). A mapping $\widetilde{M}: \mathcal{L} \rightarrow \mathcal{F}(X)$ is called a generalized fuzzy number measure, if it satisfies the following requirements:

(1) $\widetilde{M}(\phi)=\theta$;

(2) $\widetilde{M}(\cdot)$ is countably additive, i.e. for any sequence $\left(A_{n}\right)_{n=1}^{\infty}$ of pairwise disjoint elements of $\mathcal{L}$ we have that $\widetilde{M}\left(\cup_{n=1}^{\infty} A_{n}\right)=\sum_{n=1}^{\infty} \widetilde{M}\left(A_{n}\right)$, where $\sum_{n=1}^{\infty} \widetilde{M}\left(A_{n}\right)$ is defined by

$\sum_{n=1}^{\infty} \widetilde{M}\left(A_{n}\right)(x)=\sup \left\{\inf _{n} \widetilde{M}\left(A_{n}\right)\left(x_{n}\right): x=\sum_{n=1}^{\infty} x_{n}\right.$ (unconditionally convergent) $\}$. 
We recall that $M: \mathcal{L} \rightarrow 2^{X} \backslash\{\emptyset\}$ is a weak multimeasure if $s\left(x^{*}, M\right)$ is a real valued measure for all $x^{*} \in X^{*}, M: \mathcal{L} \rightarrow 2^{X} \backslash\{\emptyset\}$ is a set valued measure if it satisfies the following two requirements

(i) $M(\phi)=\{0\}$;

(ii) $M(\cdot)$ is countably additive, i.e. for any sequence $\left(A_{n}\right)_{n=1}^{\infty}$ of pairwise disjoint elements of $\mathcal{L}$ we have that $M\left(\cup_{n=1}^{\infty} A_{n}\right)=\sum_{n=1}^{\infty} M\left(A_{n}\right)$.

Each set-valued measure is a weak multimeasure. If $M$ is a weak multimeasure taking values in $c w k(X)$ also the reverse implication holds (see 18, Proposition $7.4])$.

In the sequel we will use the following representation theorem (see [30, Lemma $3.3])$.

Theorem 2.3. Let $\widetilde{M}: \mathcal{L} \rightarrow \mathcal{F}(X)$ be a generalized fuzzy number measure, $M^{r}(A)=\{x \in X: \widetilde{M}(A)(x) \geq r\}, A \in \mathcal{L}, r \in(0,1]$. Then

(i) $M^{r}: \mathcal{L} \rightarrow \operatorname{cwk}(X)$ is a set valued measure, $r \in(0,1]$;

(ii) $M^{r_{2}}(A) \subseteq M^{r_{1}}(A)$, for $0 \leq r_{1} \leq r_{2} \leq 1$ and for each $A \in \mathcal{L}$;

(iii) if $\left(r_{k}\right)$ is a nondecreasing sequence converging to $r>0$ and $A \in \mathcal{L}$, then

$$
M^{r}(A)=\bigcap_{k \geq 1} M^{r_{k}}(A) .
$$

Conversely, if there is a family $\left\{M^{r}: r \in(0,1]\right\}$ satisfying the requirements (i)-(iii), we define a mapping $\widetilde{M}: \mathcal{L} \rightarrow \mathcal{F}(X)$ as

$[\widetilde{M}(A)](x)=\left\{\begin{array}{lll}\sup \left\{r: x \in M^{r}(A), r \in(0,1]\right\} & \text { if } \quad\left\{r: x \in M^{r}(A), r \in(0,1]\right\} \neq \phi, \\ 0 & \text { if } \quad\left\{r: x \in M^{r}(A), r \in(0,1]\right\}=\phi\end{array}\right.$

Then, $\widetilde{M}$ is a generalized fuzzy number measure and $[\widetilde{M}(A)]^{r}=M^{r}(A)$ for each $A \in \mathcal{L}$ and $r \in(0,1]$.

We recall now some definitions concerning set-valued functions. A set-valued function $\Gamma: \Omega \rightarrow c w k(X)$ is said to be scalarly measurable if for every $x^{*} \in X^{*}$, the map $s\left(x^{*}, \Gamma\right)$ is measurable. A set-valued function $\Gamma: \Omega \rightarrow c w k(X)$ is said to be scalarly integrable on $\Omega$ if for each $x^{*} \in X^{*}$ the real function $s\left(x^{*}, \Gamma\right)$ is integrable on $\Omega$.

Definition 2.4. (see [15, Definition 3.1]) A set-valued function $\Gamma: \Omega \rightarrow c w k(X)$ is said to be Pettis integrable on $\Omega$ if $\Gamma$ is scalarly integrable on $\Omega$ and for each $A \in \mathcal{L}$ there exists a set $W_{\Gamma}(A) \in \operatorname{cwk}(X)$ such that for each $x^{*} \in X^{*}$, we have

$$
s\left(x^{*}, W_{\Gamma}(A)\right)=\int_{A} s\left(x^{*}, \Gamma\right) d \mu .
$$

Then we set $(P) \int_{A} \Gamma d \mu=: W_{\Gamma}(A)$, for each $A \in \mathcal{L}$. 
Observe that $W_{\Gamma}$ is a $\mu$-continuous weak multimeasure.

A function $f: \Omega \rightarrow X$ is called a selection of a set-valued function $\Gamma: \Omega \rightarrow$ $\operatorname{cwk}(X)$ if, for every $\omega \in \Omega$, one has $f(\omega) \in \Gamma(\omega)$. If $\Gamma: \Omega \rightarrow \operatorname{cwk}(X)$ is setvalued function, by the symbol $\mathcal{S}(\Gamma)$ we denote the family of all Pettis integrable selections of $\Gamma$. From now on if $\widetilde{\Gamma}: \Omega \rightarrow \mathcal{F}(X)$ is a fuzzy mapping, we set

$$
\widetilde{\Gamma}_{r}(\omega):=[\widetilde{\Gamma}(\omega)]^{r} .
$$

Given a vector valued function $f: \Omega \rightarrow X$ we call fuzzy-number valued function associated to $f$ the fuzzy mapping $\tilde{f}: \Omega \rightarrow \mathcal{F}(X)$ defined by $\widetilde{f}(\omega):=\chi_{f(\omega)}$. Using the same terminology as for multifunctions, if a function $f: \Omega \rightarrow X$ is such that $f(\omega) \in \widetilde{\Gamma}_{r}(\omega)$, for each $r \in(0,1]$, we say that the associated fuzzy mapping $\widetilde{f}(\omega)$ is a selection of $\widetilde{\Gamma}$. We recall that a fuzzy mapping $\widetilde{\Gamma}$ is said to be scalarly integrable on $\Omega$ if for all $r \in(0,1]$ the set-valued function $\widetilde{\Gamma}_{r}: \Omega \rightarrow c w k(X)$ is scalarly integrable.

\section{Fuzzy Pettis integral}

We start with the definition of fuzzy Pettis integral.

Definition 3.1. A fuzzy mapping $\widetilde{\Gamma}: \Omega \rightarrow \mathcal{F}(X)$ is said to be weakly Pettis integrable on $\Omega$ if for every $r \in(0,1]$ the set-valued function $\widetilde{\Gamma}_{r}$ is Pettis integrable on $\Omega$.

Definition 3.2. A fuzzy mapping $\widetilde{\Gamma}: \Omega \rightarrow \mathcal{F}(X)$ is said to be Pettis integrable on $\Omega$ if it is weakly Pettis integrable and, for each $A \in \mathcal{L}$, there exists a generalized fuzzy number $\widetilde{M_{\Gamma}}(A) \in \mathcal{F}(X)$ such that for any $r \in(0,1]$ and for any $x^{*} \in X^{*}$ we have

$$
s\left(x^{*},\left[\widetilde{M_{\Gamma}}(A)\right]^{r}\right)=\int_{A} s\left(x^{*}, \widetilde{\Gamma}_{r}\right) d \mu,
$$

(see [24, Definition 3.2]).

We call the fuzzy mapping $\widetilde{M_{\Gamma}}: \mathcal{L} \rightarrow \mathcal{F}(X)$ the fuzzy Pettis integral of $\widetilde{\Gamma}$ and we set $(P) \int_{A} \widetilde{\Gamma} d \mu:=\widetilde{M}_{\Gamma}(A)$.

It follows at once from the definition that a vector valued function $f: \Omega \rightarrow X$ is Pettis integrable if and only if the associated fuzzy mapping $\widetilde{f}: \Omega \rightarrow \mathcal{F}(X)$ is so. We recall the following properties:

Proposition 3.3. (see [24, Theorem 3.3]) Let $\widetilde{F}: \Omega \rightarrow \mathcal{F}(X)$ and $\widetilde{G}: \Omega \rightarrow$ $\mathcal{F}(X)$ be Pettis integrable and let $\lambda \geq 0$. Then

(1) $\widetilde{F}+\widetilde{G}$ is Pettis integrable and for each $A \in \mathcal{L}$

$$
(P) \int_{A}(\widetilde{F}+\widetilde{G}) d \mu=(P) \int_{A} \widetilde{F} d \mu+(P) \int_{A} \widetilde{G} d \mu ;
$$


(2) $\lambda \widetilde{F}$ is Pettis integrable and for each $A \in \mathcal{L}$

$$
\text { (P) } \int_{A} \lambda \widetilde{F} d \mu=\lambda(P) \int_{A} \widetilde{F} d \mu .
$$

The following decomposition result will be useful in our investigation.

Theorem 3.4. Let $\widetilde{\Gamma}: \Omega \rightarrow \mathcal{F}(X)$ be a fuzzy scalarly integrable mapping. Then, the following conditions are equivalent:

(i) $\widetilde{\Gamma}$ is fuzzy Pettis integrable on $\Omega$;

(ii) $\mathcal{S}\left(\widetilde{\Gamma}_{1}\right) \neq \emptyset$ and for every $f \in \mathcal{S}\left(\widetilde{\Gamma}_{1}\right)$, there exists a fuzzy Pettis integrable mapping $\widetilde{G}: \Omega \rightarrow \mathcal{F}(X)$ such that $\widetilde{\Gamma}(\omega)=\widetilde{G}(\omega)+\widetilde{f}(\omega)$ (where $\widetilde{f}$ is the fuzzy mapping associated to $f$ ) and, for each $r \in(0,1]$ and for each $\omega \in \Omega, 0 \in \widetilde{G}_{r}(\omega)$.

Proof. If $\widetilde{\Gamma}$ is a Pettis integrable fuzzy mapping on $\Omega$, for each $r \in(0,1]$ the multifunction $\widetilde{\Gamma}_{r}$ is Pettis integrable. Therefore $\mathcal{S}\left(\widetilde{\Gamma_{1}}\right) \neq \emptyset$ (see 8$]$ or [22]).

Let us fix $f \in \mathcal{S}\left(\widetilde{\Gamma}_{1}\right)$ and let $\widetilde{f}$ be the fuzzy-number valued function associated to $f$. Now define $\widetilde{G}: \Omega \rightarrow \mathcal{F}(X)$ setting $\widetilde{G}_{r}(\omega):=\widetilde{\Gamma}_{r}(\omega)-f(\omega)$ for each $r \in(0,1]$. At first we observe that $\widetilde{G}$ is a fuzzy mapping. In fact, since the sets $\widetilde{G}_{r}(\omega)$ are translations of the sets $\widetilde{\Gamma}_{r}(\omega)$ with a same vector, conditions $(i)-(i i i)$ of Theorem 2.1 are trivially satisfied.

We are going to prove that $\widetilde{G}$ is Pettis integrable on $\Omega$. Since for each $r \in(0,1]$, $\widetilde{\Gamma}_{r}$ is Pettis integrable and $f$ is Pettis integrable, then also $\widetilde{G}_{r}$ is Pettis integrable for each $r \in(0,1]$.

Moreover denote by $F$ the Pettis primitive of $f$ and by $\widetilde{M_{\Gamma}}$ the fuzzy Pettis integral of $\widetilde{\Gamma}$. Now for each $A \in \mathcal{L}$ define $\widetilde{N_{G}}(A)$ setting

$$
\left[\widetilde{N_{G}}(A)\right]^{r}:=\left[\widetilde{M_{\Gamma}}(A)\right]^{r}-F(A)
$$

for each $r \in(0,1]$.

Also in this case we have that $\widetilde{N_{G}}(A)$ is a fuzzy number. Moreover for each $x^{*} \in X^{*}$ and for each $r \in(0,1]$ we have

$$
\begin{aligned}
s\left(x^{*},\left[\widetilde{N_{G}}(A)\right]^{r}\right) & =s\left(x^{*},\left[\widetilde{M_{\Gamma}}(A)\right]^{r}\right)-x^{*}(F(A))=\int_{A} s\left(x^{*}, \widetilde{\Gamma}_{r}\right) d \mu-\int_{A} x^{*} f d \mu \\
& =\int_{A} s\left(x^{*}, \widetilde{\Gamma}_{r}-f\right) d \mu=\int_{A} s\left(x^{*}, \widetilde{G}_{r}\right) d \mu .
\end{aligned}
$$

Therefore $\widetilde{G}$ is Pettis is integrable. Since for each $r \in(0,1]$ and for each $\omega \in \Omega$, $\widetilde{\Gamma}_{1}(\omega) \subset \widetilde{\Gamma}_{r}(\omega)$, then $f \in \mathcal{S}\left(\widetilde{\Gamma}_{r}\right)$. Therefore $0 \in \widetilde{G}_{r}(\omega)$.

$($ ii $) \Longrightarrow($ i) follows from (1) of Proposition (3.3). 
Observe that $0 \in \widetilde{G}_{r}(\omega)$ implies that the support function $s\left(x^{*}, \widetilde{G}_{r}\right)$ is nonnegative.

We are going to prove that the fuzzy Pettis integral is a generalized fuzzy number measure.

Theorem 3.5. Let $\widetilde{\Gamma}: \Omega \rightarrow \mathcal{F}(X)$ be a Pettis integrable fuzzy mapping on $\Omega$. Then the fuzzy Pettis integral $\widetilde{M_{\Gamma}}$ of $\widetilde{\Gamma}$ is a generalized fuzzy number measure.

Proof. If $\widetilde{\Gamma}$ is a Pettis integrable fuzzy mapping, its Pettis integral is a fuzzy mapping $\widetilde{M_{\Gamma}}: \mathcal{L} \rightarrow \mathcal{F}(X)$. Moreover for each $r \in(0,1]$, the multifunction $\widetilde{\Gamma}_{r}: \Omega \rightarrow \operatorname{cwk}(X)$ is Pettis integrable and, for each $A \in \mathcal{L}$,

$$
\left[\widetilde{M_{\Gamma}}(A)\right]^{r}=(P) \int_{A} \widetilde{\Gamma}_{r} d \mu .
$$

Therefore $\left[\widetilde{M_{\Gamma}}\right]^{r}$ is a weak multimeasure. Since $\left[\widetilde{M_{\Gamma}}\right]^{r}$ takes values in $\operatorname{cwk}(X)$, it is a multimeasure, and property (i) of Theorem 2.3 is satisfied.

Moreover properties (ii) and (iii) of Theorem 2.3 are satisfied since, for each $A \in \mathcal{L}, \widetilde{M_{\Gamma}}(A)$ is a fuzzy number. Therefore the family $\left\{\left[\widetilde{M_{\Gamma}}(A)\right]^{r}: r \in(0,1]\right\}$ satisfies condition (i)-(iii) of Theorem 2.3. Then the mapping $\widetilde{M}: \mathcal{L} \rightarrow \mathcal{F}(X)$ defined as in (11) is a fuzzy number measure with $\widetilde{M}^{r}(A)=\left[\widetilde{M_{\Gamma}}(A)\right]^{r}$ for each $A \in \mathcal{L}$ and $r \in(0,1]$. Moreover $\widetilde{M}(A)=\widetilde{M_{\Gamma}}(A)$ and the assertion follows.

Let $\widetilde{\Gamma}: \Omega \rightarrow \mathcal{F}(X)$ be a Pettis integrable fuzzy mapping. Then for each $r \in(0,1]$, $\widetilde{\Gamma}_{r}: \Omega \rightarrow c w k(X)$ is a Pettis integrable multifunction. Therefore (see [22, Theorem 1.4]), for each $r \in(0,1]$, the operator $T_{r}: X^{*} \rightarrow L^{1}(\mu)$, defined as $T_{r}\left(x^{*}\right):=s\left(x^{*}, \widetilde{\Gamma}_{r}\right)$ is $\tau\left(X^{*}, X\right)$ - weakly continuous, where $\tau\left(X^{*}, X\right)$ denotes the topology of uniform convergence on weakly compact subsets of $X$.

In case of fuzzy mappings, we have the following characterization.

Theorem 3.6. Let $\widetilde{\Gamma}: \Omega \rightarrow \mathcal{F}(X)$ be a scalarly integrable fuzzy mapping. Then $\widetilde{\Gamma}$ is Pettis integrable if and only if for each $r \in(0,1]$ the operator $T_{r}$ is $\tau\left(X^{*}, X\right)$ weakly continuous. If $0 \in \widetilde{\Gamma}_{r}(\omega)$ for each $r \in(0,1]$ and for each $\omega \in \Omega$, then $T_{r}$ is $\tau\left(X^{*}, X\right)$-norm continuous.

Proof. Let $\widetilde{\Gamma}: \Omega \rightarrow \mathcal{F}(X)$ be a Pettis integrable fuzzy mapping. Then for each $r \in(0,1], \widetilde{\Gamma}_{r}: \Omega \rightarrow \operatorname{cwk}(X)$ is a Pettis integrable multifunction therefore by [22, Theorem 1.4] the operator $T_{r}$ is $\tau\left(X^{*}, X\right)$-weakly continuous.

Conversely assume that for each $r \in(0,1]$ the operator $T_{r}$ is $\tau\left(X^{*}, X\right)$-weakly continuous. Again by [22, Theorem 1.4] it follows that $\widetilde{\Gamma}_{r}: \Omega \rightarrow \operatorname{cwk}(X)$ is a Pettis integrable multifunction. For each $A \in \mathcal{L}$ and for each $r \in(0,1]$ define 
$\left[\widetilde{M_{\Gamma}}(A)\right]^{r} \in \operatorname{cwk}(X)$ such that

$$
s\left(x^{*},\left[\widetilde{M_{\Gamma}}(A)\right]^{r}\right)=\int_{A} s\left(x^{*}, \widetilde{\Gamma}_{r}\right) d \mu .
$$

We have to prove that for any $A \in \mathcal{L}$ the family $\left\{\left[\widetilde{M_{\Gamma}}(A)\right]^{r}: r \in(0,1]\right\}$, satisfies properties (i)-(iii) of Theorem 2.1. Clearly condition (i) is satisfied. In order to prove (ii), let $0 \leq r_{1} \leq r_{2} \leq 1$. Since $\widetilde{\Gamma}$ is a fuzzy mapping, by Theorem 2.1 we have $\widetilde{\Gamma}_{r_{2}}(\omega) \subseteq \widetilde{\Gamma}_{r_{1}}(\omega)$, for each $\omega \in \Omega$. Therefore

$$
s\left(x^{*}, \widetilde{\Gamma}_{r_{2}}\right) \leq s\left(x^{*}, \widetilde{\Gamma}_{r_{1}}\right),
$$

for each $x^{*} \in X^{*}$. Therefore for each $A \in \mathcal{L}$ and for each $x^{*} \in X^{*}$

$$
s\left(x^{*},\left[\widetilde{M_{\Gamma}}(A)\right]^{r_{2}}\right)=\int_{A} s\left(x^{*}, \widetilde{\Gamma}_{r_{2}}\right) d \mu \leq \int_{A} s\left(x^{*}, \widetilde{\Gamma}_{r_{1}}\right) d \mu=s\left(x^{*},\left[\widetilde{M_{\Gamma}}(A)\right]^{r_{1}}\right) .
$$

Then, as a consequence of Hahn-Banach separation theorem for convex sets, by (3) we also infer the inclusion $\left[\widetilde{M_{\Gamma}}(A)\right]^{r_{2}} \subseteq\left[\widetilde{M_{\Gamma}}(A)\right]^{r_{1}}$ for each $A \in \mathcal{L}$ and property (ii) is satisfied. If $\left(r_{k}\right)$ is a nondecreasing sequence converging to $r>0$, then for each $\omega \in \Omega$ we have

$$
\widetilde{\Gamma}_{r}(\omega)=\bigcap_{k \geq 1} \widetilde{\Gamma}_{r_{k}}(\omega) .
$$

Consequently (see [26, Proposition 1])

$$
s\left(x^{*}, \widetilde{\Gamma}_{r}\right)=\lim _{k} s\left(x^{*}, \widetilde{\Gamma}_{r_{k}}\right),
$$

for each $\omega \in \Omega$ and $x^{*} \in X^{*}$.

By hypothesis, for each $x^{*} \in X$, the sequence of real valued functions $\left(s\left(x^{*}, \widetilde{\Gamma}_{r_{k}}\right)\right)$ is integrable. As in Theorem [3.4 we can observe that, for each $r \in(0,1]$ and for each $\omega \in \Omega, 0 \in \widetilde{G}_{r}(\omega)=\widetilde{\Gamma}_{r}(\omega)-f(\omega)$. Then, without loss of generality, we can assume that $s\left(x^{*}, \widetilde{\Gamma}_{r_{k}}\right) \geq 0$. Since $s\left(x^{*}, \widetilde{\Gamma}_{r_{1}}\right) \geq s\left(x^{*}, \widetilde{\Gamma}_{r_{k}} \geq 0\right.$, by Lebesgue dominated convergence theorem we get

$$
\begin{aligned}
s\left(x^{*},\left[\widetilde{M_{\Gamma}}(A)\right]^{r}\right) & =\int_{A} s\left(x^{*}, \widetilde{\Gamma}_{r}\right) d \mu \\
& =\lim _{k} \int_{A} s\left(x^{*}, \widetilde{\Gamma}_{r_{k}}\right) d \mu \\
& =\lim _{k} s\left(x^{*},\left[\widetilde{M_{\Gamma}}(A)\right]^{r_{k}}\right)=s\left(x^{*}, \bigcap_{k \geq 1}\left[\widetilde{M_{\Gamma}}(A)\right]^{r_{k}}\right) .
\end{aligned}
$$

Since above equalities hold for each $x^{*} \in X^{*}$, we obtain $\left[\widetilde{M_{\Gamma}}(A)\right]^{r}=\bigcap_{k \geq 1}\left[\widetilde{M_{\Gamma}}(A)\right]^{r_{k}}$ and property (iii) is satisfied. 
If $0 \in \widetilde{\Gamma}_{r}(\omega)$ for each $r \in(0,1]$ then again by [22, Theorem 1.4] it follows that $T_{r}$ is $\tau\left(X^{*}, X\right)$-norm continuous.

We recall that $X^{*}$ is said to be weak* - angelic if for each bounded set $B \subset X^{*}$ the weak ${ }^{*}$-closure of $B$ is equal to the set of weak*-limits of sequences from $B$.

Definition 3.7. We say that a space $Y \subset X$ determines a fuzzy mapping $\widetilde{\Gamma}: \Omega \rightarrow \mathcal{F}(X)$, if for each $r \in(0,1], s\left(x^{*}, \widetilde{\Gamma}_{r}\right)=0 \mu-$ a.e. for each $x^{*} \in Y^{\perp}$, where $Y^{\perp}$ denotes the annihilator of $Y$ in $X^{*}$ and the exceptional sets depend on $x^{*}$ and on $r$.

Theorem 3.8. Let $\widetilde{\Gamma}: \Omega \rightarrow \mathcal{F}(X)$ be a scalarly integrable fuzzy mapping determined by a space $Y \subseteq X$ such that $Y^{*}$ is weak*-angelic and such that for each $r \in(0,1]$ the operator $T_{r}: X^{*} \rightarrow L^{1}(\mu)$ is weakly compact. Then $\widetilde{\Gamma}$ is a Pettis integrable fuzzy mapping.

Proof. Let $\widetilde{\Gamma}: \Omega \rightarrow \mathcal{F}(X)$ be a scalarly integrable fuzzy mapping satisfying the hypothesis of the claim. Then for each $r \in(0,1], \widetilde{\Gamma}_{r}: \Omega \rightarrow \operatorname{cwk}(X)$ is a scalarly integrable multifunction and satisfies hypotheses (WC) and $\left(\mathrm{D}^{*}\right)$ of 22 , Theorem 2.4]. Therefore it follows that $\widetilde{\Gamma}_{r}$ is a Pettis integrable multifunction. For each $A \in \mathcal{L}$ and for each $r \in(0,1]$ let $\left[\widetilde{M_{\Gamma}}(A)\right]^{r} \in \operatorname{cwk}(X)$ be such that equality (2) holds.

We have to prove that for any $A \in \mathcal{L}$ the family $\left\{\left[\widetilde{M_{\Gamma}}(A)\right]^{r}: r \in(0,1]\right\}$ satisfies properties (i)-(iii) of Theorem 2.1. To this end it is enough to proceed as in the proof of the "only if" part of Theorem 3.6 . Thus $\widetilde{M_{\Gamma}}$ defines a fuzzy mapping and we conclude that $\widetilde{\Gamma}$ is fuzzy Pettis integrable.

Definition 3.9. A subspace $Y \subset X$ is said to be weakly compact generated (WCG) if there is a weakly compact set $K$ in $Y$ such that $Y=\overline{\operatorname{span}}(K)$.

Examples of WCG spaces are separable spaces, reflexive spaces, $L^{1}(\mu)$ if $\mu$ is a $\sigma$-finite measure, and $c_{0}(\Gamma)$ if $\Gamma$ is a nonempty set (see [17, Chapters 13]).

Proposition 3.10. Let $\widetilde{\Gamma}: \Omega \rightarrow \mathcal{F}(X)$ be a Pettis integrable fuzzy mapping. Then $\widetilde{\Gamma}$ is determined by a WCG subspace of $X$ and for each $r \in(0,1]$ the operator $T_{r}: X^{*} \rightarrow L^{1}(\mu)$ is weakly compact.

Proof. If $\widetilde{\Gamma}: \Omega \rightarrow \mathcal{F}(X)$ is Pettis integrable, then for each $r \in(0,1], \widetilde{\Gamma}_{r}$ : $\Omega \rightarrow c w k(X)$ is a Pettis integrable multifunction. Therefore by [22, Proposition $2.2]$ the operator $T_{r}: X^{*} \rightarrow L^{1}(\mu)$ is weakly compact. Moreover as in [22, Proposition 2.2], denote by $Y_{r}$ the weakly compact generated subspace of $X$ which determines the multifunction $\widetilde{\Gamma}_{r}$. If $0<r_{1} \leq r_{2} \leq 1$, then $\widetilde{\Gamma}_{r_{2}}(\omega) \subseteq$ $\widetilde{\Gamma}_{r_{1}}(\omega)$, and also $Y_{r_{2}} \subseteq Y_{r_{1}}$. Let now $\left\{s_{k}\right\}$ be a sequence in $(0,1]$ such that $s_{k} \searrow 0$ 
and for each $k$ let $W_{s_{k}} \subset B(X)$ be a weakly compact convex set generating $Y_{s_{k}}$. The set $\bigcup_{k} \frac{1}{2^{k}} W_{s_{k}}$ is a weakly compact set generating a space $Y$. So if $x^{*} \in Y^{\perp}$, for each $s_{k}, x^{*} \in Y_{s_{k}}^{\perp}$ and then also $x^{*} \in Y_{r}^{\perp}$ for each $r \in(0,1]$. It follows from 22. Proposition 2.2] that $s\left(x^{*}, \widetilde{\Gamma}_{r}\right)=0 \mu$-a.e. This ends the proof.

We recall that the class of Banach spaces having weak ${ }^{*}$-angelic dual is very large and contains all weakly compactly generated spaces (16. Chapters 1112]). Then the following theorem is a consequence the previous Theorem 3.8 and Proposition 3.10

Theorem 3.11. A scalarly integrable fuzzy mapping $\widetilde{\Gamma}: \Omega \rightarrow \mathcal{F}(X)$ is Pettis integrable if and only if $\widetilde{\Gamma}$ is determined by a WCG space $Y \subseteq X$ and for each $r \in(0,1]$ the operator $T_{r}: X^{*} \rightarrow L^{1}(\mu)$ is weakly compact.

As a consequence of Theorem 3.11 we obtain:

Corollary 3.12. Let $\widetilde{\Gamma}: \Omega \rightarrow \mathcal{F}(X)$ be a scalarly measurable fuzzy mapping. Assume that there exists a fuzzy mapping $\widetilde{G}: \Omega \rightarrow \mathcal{F}(X)$ which is Pettis integrable and satisfies, for each $x^{*} \in X^{*}$ and $r \in(0,1]$,

$$
\left|s\left(x^{*}, \widetilde{\Gamma}_{r}\right)\right| \leq\left|s\left(x^{*}, \widetilde{G}_{r}\right)\right| .
$$

Then $\widetilde{\Gamma}$ is Pettis integrable.

Proof. Indeed inequality (4) implies that for each $r \in(0,1], \widetilde{\Gamma}$ is determined by a WCG space $Y \subseteq X$ and the weak compactness of the operator $T_{r}: X^{*} \rightarrow L^{1}(\mu)$, for each $r \in(0,1]$.

It is known that if $X$ is a separable Banach space and $c_{0} \not \subset X$, then each scalarly integrable function is Pettis integrable. An analogue result, for weakly integrable bounded fuzzy mapping has been proved in [32, Theorem 4.5] in case of a separable space. In the following theorem we prove Pettis integrability of a scalarly integrable fuzzy mapping in case of a general Banach space without any boundness condition, extending [22, Theorem 2.13] proved for multifunctions.

Theorem 3.13. Let $X$ be a Banach space not containing any isomorphic copy of $c_{0}$. If $\widetilde{\Gamma}: \Omega \rightarrow \mathcal{F}(X)$ is a scalarly measurable fuzzy mapping which is determined by a WCG space, then $\widetilde{\Gamma}$ is Pettis integrable.

Proof. If $\widetilde{\Gamma}: \Omega \rightarrow \mathcal{F}(X)$ is a scalarly integrable fuzzy mapping, then for each $r \in$ $(0,1], \widetilde{\Gamma}_{r}: \Omega \rightarrow \operatorname{cwk}(X)$ is a scalarly integrable multifunction and is determined by a WCG space. Therefore by [22, Theorem 2.13] it follows that $\widetilde{\Gamma}_{r}: \Omega \rightarrow$ $c w k(X)$ is a Pettis integrable multifunction.

Let $\left[\widetilde{M}_{\Gamma}\right]^{r}$ be the Pettis integral of $\widetilde{\Gamma}_{r}$. It is enough to prove that for each $A \in \mathcal{L}$ the family $\left\{\left[\widetilde{M_{\Gamma}}(A)\right]^{r}: r \in(0,1]\right\}$, satisfies properties (i)-(iii) of Theorem 2.1 . 
So, proceeding as in the proof of the "only if" part of the proof Theorem 3.6. we get the result.

\section{Core CHARACterization}

The result of Talagrand which characterizes Pettis integrability of a vector valued function $f$ is extended to multifunction in 22 . We recall that (see 22 Definition 4.3) if $\Gamma: \Omega \rightarrow c w k(X)$ is a multifunction, for each $E \in \Sigma$, the core of $\Gamma$ on $E$ is defined by

$$
\operatorname{cor}_{\Gamma}(E)=\bigcap_{\mu(N)=0} \overline{\operatorname{conv}} \Gamma(E \backslash N)=\bigcap_{\mu(N)=0} \overline{\operatorname{conv}}\left(\bigcup_{\omega \in E \backslash N} \Gamma(\omega)\right) .
$$

We say that a fuzzy mapping $\widetilde{G}: \Omega \rightarrow \mathcal{F}(X)$ is dominated by a fuzzy mapping $\widetilde{\Gamma}: \Omega \rightarrow \mathcal{F}(X)$ if, for each $r \in(0,1]$ and for each $\omega \in \Omega, \widetilde{G}_{r}(\omega) \subseteq \widetilde{\Gamma}_{r}(\omega)$.

The following theorem extends to scalarly integrable fuzzy mappings the core characterization of Pettis integrability.

The symbol $\Sigma^{+}$denotes the sets $E \in \Sigma$ with $\mu(E)>0$.

Theorem 4.1. Let $\widetilde{\Gamma}: \Omega \rightarrow \mathcal{F}(X)$ be a scalarly integrable fuzzy mapping. Then $\widetilde{\Gamma}$ is Pettis integrable if and only if the following properties hold:

(1) for each $r \in(0,1]$ the operator $T_{r}: X^{*} \rightarrow L^{1}(\mu)$ is weakly compact;

(2) if $\widetilde{G}: \Omega \rightarrow \mathcal{F}(X)$ is a scalarly measurable fuzzy mapping that is dominated by $\widetilde{\Gamma}$, then for each $r \in(0,1], \operatorname{cor}_{\widetilde{G}_{r}}(E) \neq \emptyset$, for every $E \in \Sigma^{+}$.

Proof. Assume first that $\widetilde{\Gamma}$ is Pettis integrable, then for each $r \in(0,1]$, the multifunction $\widetilde{\Gamma}_{r}: \Omega \rightarrow \operatorname{cwk}(X)$ is Pettis integrable. Therefore the operator $T_{r}: X^{*} \rightarrow L^{1}(\mu)$ is weakly compact. Moreover if $\widetilde{G}: \Omega \rightarrow \mathcal{F}(X)$ is a scalarly measurable fuzzy mapping which is dominated by $\widetilde{\Gamma}$, we have that $\widetilde{G}_{r}(\omega) \subseteq$ $\widetilde{\Gamma}_{r}(\omega)$ for each $\omega$ and for each $r$. Then applying [22, Theorem 4.6], we get that $\operatorname{cor}_{\widetilde{G}_{r}}(E) \neq \emptyset$, for every $E \in \Sigma^{+}$.

Conversely assume that conditions (1) and (2) are satisfied. This implies that for each $r \in(0,1]$, the multifunction $\widetilde{\Gamma}_{r}: \Omega \rightarrow c w k(X)$ is a scalarly integrable multifunction, such that the operator $T_{r}: X^{*} \rightarrow L^{1}(\mu)$ is weakly compact. Moreover condition (2) implies that $\operatorname{cor}_{\widetilde{G}_{r}}(E) \neq \emptyset$, for each $r$ and for every $E \in \Sigma^{+}$. Therefore, applying again [22, Theorem 4.6], it follows that $\widetilde{\Gamma}_{r}: \Omega \rightarrow$ $\operatorname{cwk}(X)$ is a Pettis integrable multifunction.

If again $\left[\widetilde{M_{\Gamma}}\right]^{r}$ is the Pettis integral of $\widetilde{\Gamma}_{r}$, then, by proceeding as in the proof of Theorem 3.6. we infer that for each $A \in \mathcal{L}$ the family $\left\{\left[\widetilde{M_{\Gamma}}(A)\right]^{r}: r \in(0,1]\right\}$, satisfies properties (i)-(iii) of Theorem 2.1, Therefore the assertion follows. 
Observe that if $\widetilde{\Gamma}: \Omega \rightarrow \mathcal{F}(X)$ is a Pettis integrable fuzzy mapping, then each multifunction $\widetilde{\Gamma}_{r}: \Omega \rightarrow \operatorname{cwk}(X)$ possesses Pettis integrable selections (see [8, Theorem 2.6]). As a consequence of previous theorem we obtain that

Theorem 4.2. Let $\widetilde{\Gamma}: \Omega \rightarrow \mathcal{F}(X)$ be a scalarly integrable fuzzy mapping. If for each $r \in(0,1]$ the operator $T_{r}: X^{*} \rightarrow L^{1}(\mu)$ is weakly compact and every scalarly measurable selection of $\widetilde{\Gamma}_{r}$ is Pettis integrable, then $\widetilde{\Gamma}$ is a Pettis integrable fuzzy mapping.

Proof. Let $\widetilde{G}: \Omega \rightarrow \mathcal{F}(X)$ be any scalarly measurable fuzzy mapping that is dominated by $\widetilde{\Gamma}$. Then, for each $r \in(0,1]$, the multifunction $\widetilde{G}_{r}$ has scalarly measurable selections (see [9]). It follows by the hypothesis that each scalarly measurable selection of $\widetilde{G}_{r}$ is Pettis integrable. Therefore $\operatorname{cor}_{\widetilde{G}_{r}}(E) \neq \emptyset$, for every $E \in \Sigma^{+}$. Consequently by Theorem 4.1 , we get that $\widetilde{\Gamma}$ is a Pettis integrable fuzzy mapping.

ACKNOWLEDGMENTs. The authors are grateful to the anonymous reviewers for their valuable remarks.

\section{REFERENCES}

[1] E.J. BALDER, - A.R. SAMBUCINI, On weak compactness and lower closure results for Pettis integrable (multi)function, Bull. Pol. Acad. Sci. 52, 1, (2004), 53-61.

[2] A. BOCCUTO, -A.R. SAMBUCINI, A note on comparison between Birkhoff and McShane-type integrals for multifunctions, Real Analysis Exchange, 37(2), (2011/2012), 315-324.

[3] A. BOCCUTO,- D. CANDELORO,-A. R. SAMBUCINI Henstock multivalued integrability in Banach lattices with respect to pointwise non atomic measures, Atti Accad. Naz. Lincei Rend. Lincei Mat. Appl. 26, (2015), 363-383.

[4] B. BONGIORNO, - L. DI PIAZZA, - K. MUSIAL, A decomposition theorem for the fuzzy Henstock integral, Fuzzy sets and Systems,200 (2012), 36-47.

[5] D. CANDELORO,-- L. DI PIAZZA, -K. MUSIAL, -A.R. SAMBUCINI Gauge integrals and selections of weakly compact valued multifunctions, Journal of Mathematical Analysis and Applications, 441, (2016), 293308.

[6] C. CASTAING, - M. VALADIER, Convex Analysis and Measurable Multifunctions, Lect. Notes Math., 580, Springer-Verlag, Berlin 1977.

[7] B. CASCALES, -V. KADETS, - J. RODRIGUEZ, The Pettis integral for multi-valued functions via single-valued ones, J. Math. Anal. Appl. 332, (1), (2007), 1-10.

[8] B. CASCALES, -V. KADETS, - J. RODRIGUEZ, Measurable selectors and set-valued Pettis integral in nonseparable Banach spaces, J. Funct. Anal., 256, (3), (2009), 673-699.

[9] B. CASCALES, -V. KADETS, - J. RODRIGUEZ, Measurability and selections of multifunctions in Banach spaces, J. Convex Anal., 17, (1), (2010), 229-240.

[10] B. CASCALES, - J. RODRIGUEZ, Birkhoff integral for multi-valued functions, J. Math. Anal. Appl., 297, (2), (2004), 540-560, Special issue dedicated to John Horvaàrt.

[11] P. DIAMOND, - P. KLOEDEN, Characterization of compact subsets of fuzzy sets, Fuzzy Sets and Systems 29 (1989), no. 3, 341-348. 
[12] L. DI PIAZZA, -K. MUSIAL, Set-valued Kurzweil-Henstock-Pettis integral, Set-Valued Analysis 13, (2005), 167-179.

[13] L. DI PIAZZA, -K. MUSIAL, A decomposition theorem for compact-valued Henstock integral, Monatsh. Math. 148 (2), (2006), 119-126.

[14] L. DI PIAZZA, -K. MUSIAL, A decomposition of Henstock-Kurzweil-Pettis integrable multifunctions, Vector Measures, Integration and Related Topics (Eds.) G.P. Curbera, G. Mockenhaupt, W.J. Ricker, Operator Theory: Advances and Applications Vol. 201 (2010) pp. 171-182 Birkhauser Verlag, ISBN: 978-3-0346-0210-5 Hardcover.

[15] K. EL AMRI, -C. HESS, On the Pettis integral of closed valued multifunctions, SetValued Anal. 8 (2000), 329-360.

[16] M. FABIAN, -P. HABALA, -P. HAJEK, - V. MONTESINOS SANTALUCA, -J. PELANT, - V. ZIZLER, Functional Analysis and Infinite-dimensional Geometry, CMS Books in Mathematics/Ouvrages de Mathematiques de la SMC, vol. 8, Springer-Verlag, New York, 2001.

[17] M. FABIAN, -P. HABALA, -P. HAJEK,-V. MONTESINOS, -V. ZIZLER, Banach Space Theory- The Basis for Linear and Nonlinear Analysis, CMS Books in Mathematics/Canadian Mathematical Society, Springer-Verlag, New York, 2011.

[18] S. HU, - N.S. PAPAGEORGIOU, Handbook of Multivalued Analysis. Vol. I: Theory, Kluwer Academic Publishers (1997).

[19] O. KALEVA, Fuzzy integral equations, Fuzzy sets and Systems, 24 (1987), 301-317.

[20] A. MARTELlOTTI, -A.R. SAMBUCINI, On the comparison between Aumann and Bochner integral, Journal of Mathematical Analysis and Applications, 260, No. 1, (2001), 6-17.

[21] M. MATLOKA, On fuzzy integral, Proc. Polish Symp., Interval and Fuzzy Math. 1989, Poznan 163-170.

[22] K. MUSIAL, Pettis integration of multifunctions with values in arbitrary Banach spaces, J. Convex Analysis, 18, (2011),769-810.

[23] K. MUSIAL, A decomposition theorem for Banach space valued fuzzy Henstock integral, Fuzzy sets and Systems, 259, (2015), 21-28.

[24] CHUN-KEE PARK, On the Pettis integral of fuzzy mappings in Banach spaces, Commun. Korean Math. Soc. 22 (2007), 535-545.

[25] CHUN-KEE PARK, On the Birkhoff integral of fuzzy mappings in Banach spaces, Korean J. Math. Soc. 21 (2013), 439-454.

[26] Y. SONNTAG, Scalar Convergence of Convex Sets, JMAA , 164 (1992), 219-241.

[27] C. WU, - Z. GONG, On Henstock integrals of interval-valued and fuzzy-number-valued functions, Fuzzy Sets and Systems 115 (2000), 377-391.

[28] C. WU,-Z. GONG, On Henstock integrals of fuzzy-valued functions (I), Fuzzy Sets and Systems 120 (2001), 523-532.

[29] C. WU, - M. MA,- J. FANG, Structure theory of fuzzy analysis, Guizhou Scientific publication, Guiyang, China (1994).

[30] J. WU, -X. XUE, - C. WU, Radon-Nikodym theorem and Vitali-Hahn-Saks theorem on fuzzy number measures in Banach spaces, Fuzzy Sets and Systems, 117, (2001), 339-346.

[31] J. WU, - C. WU, The w-derivatives of fuzzy mappings in Banach spaces, Fuzzy sets and systems, 119, (2001), 375-381.

[32] XUE XIAOPING, -HA MINGHU ,- MA MING, Random fuzzy number integrals in Banach spaces, Fuzzy sets and systems, 66, (1994), 97-111.

[33] XUE XIAOPING, -HA MINGHU, -WU CONGXIN, On the extension of the fuzzy number measures in Banach spaces: Part I. Representation of the fuzzy number measures, Fuzzy sets and systems, 78, (1996), 347-354. 
[34] XUE XIAOPING, - WANG, X., -WU,L., On the convergence and representation of random fuzzy number integrals, Fuzzy sets and systems, 103, (1999), 115-125.

* Department of Mathematics and Computer Sciences

University of Palermo

VIA Archirafi 34

90123 PALERMo

ITALY

E-mail address: luisa.dipiazza@unipa.it

* Department of Mathematics and Computer Sciences University of Palermo

VIA ArChirafi 34

90123 PALERMo

ITALY

E-mail address: valeria.marraffa@unipa.it 